\title{
A measurement method of material parameters for uniaxially anisotropic artificial dielectrics
}

\author{
Toshio Ishizaki $^{1 a)}$, Seiji Kida ${ }^{2}$, and Ikuo Awai ${ }^{2}$ \\ ${ }^{1}$ Panasonic Corporation \\ 1 Kotari-yakemachi, Nagaokakyo, Kyoto 617-8520, Japan \\ ${ }^{2}$ Ryukoku University, \\ 1-5 Yokotani, Seta-oecho, Otsu, Shiga, 520-2194 Japan \\ a) ishizaki.toshio@jp.panasonic.com
}

\begin{abstract}
A novel measurement method of material parameters for uniaxially anisotropic artificial dielectrics is proposed. The method utilizes the transmission characteristics with a waveguide. This method is valid for the artificial dielectrics where the size of constituting particles is negligibly small compared with the wavelength, and the frequency dependency of the material parameters is very small. Our method requires only one piece of sample for measurement. In the case where the test piece of the material is thin enough, the material parameters can be calculated analytically. The theoretical considerations are confirmed by the experiments for artificial dielectrics.
\end{abstract}

Keywords: artificial dielectrics, material parameters, uniaxially anisotropic, measurement method, transmission characteristics

Classification: Microwave and millimeter wave devices, circuits, and systems

\section{References}

[1] I. Awai, O. Mizue, and A. K. Saha, "Artificial Dielectric Resonator Made of Spherical Metal Particles," IEICE Trans. Electron., vol. E92-C, no. 1, pp. 72-76, Jan. 2009.

[2] S. Kida and I. Awai, "Measurement Method of Uniaxially Anisotropic Material Constants," IEICE Electron. Society Conf. 2009, C-2-68, 2009 (In Japanese).

\section{Introduction}

Recently, artificial dielectric is one of the hot topics, because the unfamiliar nature of the materials is very useful for various applications. The material parameters for isotropic artificial dielectric are analyzed by computer simulations and measured by waveguide methods [1]. However, there is some 
difficulty for uniaxially anisotropic materials that is very common anisotropy in artificial dielectrics. The authors propose a new idea for measuring the material parameters, and then, confirm it with simulation and experiment. This method is valid for the artificial dielectrics where the size of constituting particles is negligibly small compared with the wavelength, and the frequency dependency of the material parameters is very small. The calculation equations are derived in this paper, being compared with the two-waveguide method.

\section{Fundamental equations}

The material parameters of uniaxial anisotropy in the z-direction are considered. If the size of constituting particle is far smaller than the wavelength, the permittivity and the permeability are defined as follows due to the common knowledge of Crystallography.

$$
\begin{aligned}
& {[\varepsilon]=\left[\begin{array}{ccc}
\varepsilon_{t} & 0 & 0 \\
0 & \varepsilon_{t} & 0 \\
0 & 0 & \varepsilon_{z}
\end{array}\right]} \\
& {[\mu]=\left[\begin{array}{ccc}
\mu_{t} & 0 & 0 \\
0 & \mu_{t} & 0 \\
0 & 0 & \mu_{z}
\end{array}\right]}
\end{aligned}
$$

The setup for measurement and sample of artificial dielectrics are shown in Fig. 1. Metal particles (colored in Fig. 1(b)) act as natural molecules. Thus, the permittivity and the permeability can be tailored by the particle size and cell structure.

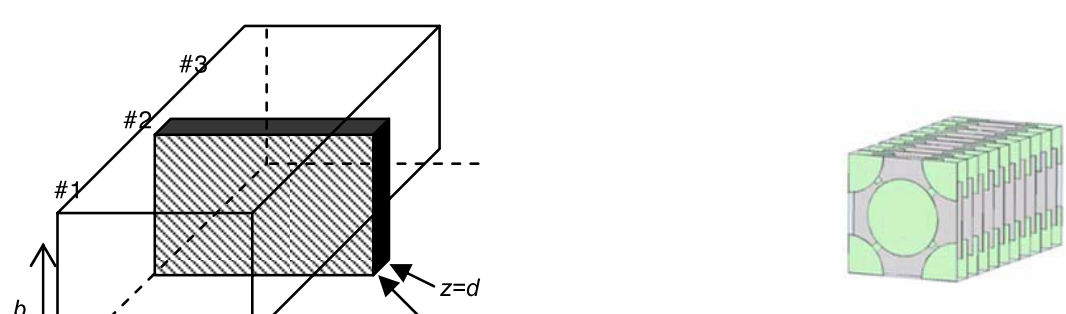

(b) Unit cells of the sample with FaceCentered Tetragonal (FCT) structure

(a) Setup for measurement

Fig. 1. The setup for measurement and unit cells of DUT with Face-Centered Tetragonal (FCT) structure

For $\mathrm{TE}_{10}$-mode, the characteristic impedance and the propagation constant in the material (\#2) are expressed as

$$
Z_{w}=\frac{j \omega \mu_{t}}{\gamma}
$$




$$
\gamma=\sqrt{\left(\frac{\pi}{a}\right)^{2} \cdot \frac{\mu_{t}}{\mu_{z}}-\omega^{2} \varepsilon_{t} \mu_{t}} .
$$

In regions \#1 and \#3, the normalized field distribution is

$$
\begin{gathered}
E_{y}=\sin \left(\frac{\pi}{a} x\right) \cdot e^{ \pm j \beta z} \\
H_{x}=-Z_{o} \sin \left(\frac{\pi}{a} x\right) \cdot e^{ \pm j \beta z}
\end{gathered}
$$

where

$$
\begin{gathered}
\beta=\sqrt{\omega^{2} \varepsilon_{o} \mu_{o}-\left(\frac{\pi}{a}\right)^{2}} \\
Z_{o}=\frac{\omega \mu_{o}}{\beta}
\end{gathered}
$$

Now, considering Maxwell's equation in region \#2, the field distribution along z-axis in region \#2 is described by $e^{-\gamma z}$ and written as

$$
\begin{gathered}
-\gamma E_{y}=j \omega \mu_{t} H_{x} \\
\gamma E_{x}=-j \omega \mu_{t} H_{y} \\
\frac{\partial H_{z}}{\partial x}=-\frac{\gamma^{2}+\omega^{2} \varepsilon_{t} \mu_{t}}{\gamma} \cdot H_{x} \\
\frac{\partial H_{z}}{\partial y}=-\frac{\gamma^{2}+\omega^{2} \varepsilon_{t} \mu_{t}}{\gamma} \cdot H_{y}
\end{gathered}
$$

In region $\# 2$, $\mathrm{TE}_{10}$-mode is propagating.

In region $\# 1$, the horizontal field distributions are expressed as

$$
\begin{gathered}
E_{y}=\left(A e^{-j \beta z}+B e^{j \beta z}\right) \cdot \sin \left(\frac{\pi x}{a}\right) \\
H_{x}=-\frac{1}{Z_{o}}\left(A e^{-j \beta z}-B e^{j \beta z}\right) \cdot \sin \left(\frac{\pi x}{a}\right)
\end{gathered}
$$

In region $\# 2$,

$$
\begin{gathered}
E_{y}=\left(C e^{-\gamma z}+D e^{\gamma z}\right) \cdot \sin \left(\frac{\pi x}{a}\right) \\
H_{x}=-\frac{1}{Z_{w}}\left(C e^{-\gamma z}-D e^{\gamma z}\right) \cdot \sin \left(\frac{\pi x}{a}\right)
\end{gathered}
$$

In region \#3,

$$
\begin{gathered}
E_{y}=E e^{-j \beta(z-d)} \cdot \sin \left(\frac{\pi x}{a}\right) \\
H_{x}=-\frac{E}{Z_{o}} e^{-j \beta(z-d)} \cdot \sin \left(\frac{\pi x}{a}\right)
\end{gathered}
$$

Due to the continuity conditions for $E_{y}$ and $H_{x}$ at the two boundaries: $z=0$ and $d$, the following relations are obtained,

$$
\begin{aligned}
& S_{21}=\frac{E}{A}=\frac{-4 \kappa \eta}{(\kappa-1)^{2} \eta^{2}-(\kappa+1)^{2}} \\
& S_{11}=\frac{B}{A}=\frac{\left(\kappa^{2}-1\right) \cdot\left(\eta^{2}-1\right)}{(\kappa-1)^{2} \eta^{2}-(\kappa+1)^{2}},
\end{aligned}
$$

where

$$
\kappa=\frac{Z_{w}}{Z_{o}}=\frac{j \beta \mu_{t}}{\gamma \mu_{o}}
$$




$$
\eta=e^{-\gamma d}
$$

Finally, the following equations are obtained after rather tedious manipulation.

$$
\begin{gathered}
\kappa=\sqrt{\frac{\left(1+S_{11}\right)^{2}-S_{21}^{2}}{\left(1-S_{11}\right)^{2}-S_{21}^{2}}} \\
\gamma=\frac{1}{d} \cdot \cosh ^{-1} \frac{1-S_{11}^{2}+S_{21}^{2}}{2 S_{21}}
\end{gathered}
$$

These two equations determine $\kappa$ and $\gamma$ by the measured S-parameters, $S_{11}$ and $S_{21}$. Therefore, $\mu_{t}$ is obtained from equation (21) as

$$
\mu_{t}=j \frac{\gamma \kappa \mu_{o}}{\beta}
$$

Since $\gamma$ contains $\varepsilon_{t}$ and $\mu_{z}$ at the same time as shown in eq. (4), $\mu_{z}$ and $\varepsilon_{t}$ cannot be separated only from the value of $\gamma$. Therefore some new idea is required. We now define the parameter $F$ as

$$
F \equiv\left\{\left(\frac{\pi}{a}\right)^{2} \cdot \frac{1}{\mu_{z}}-\omega^{2} \varepsilon_{t}\right\}=\frac{\gamma^{2}}{\mu_{t}}
$$

$F$ can be calculated from the measured $\gamma$ and $\mu_{t}$. At this moment, $\mu_{t}$ and $F$ is determined as a function of angular frequency.

\section{Separation of parameters}

As mentioned above, we can obtain only two values out of three unknown parameters, $\mu_{t}, \mu_{z}$ and $\varepsilon_{t}$. Here, the partial differentials of the two values regarding angular frequency are calculated to separate the three parameters.

The quantities $\mu$ and $\varepsilon$ could be assumed to be frequency independent considering the movement of electrons in metal particles. Then, the following approximation equations are obtained.

$$
\begin{aligned}
& \frac{\partial \mu_{t}}{\partial \omega} \approx 0 \\
& \frac{\partial \mu_{z}}{\partial \omega} \approx 0 \\
& \frac{\partial \varepsilon_{t}}{\partial \omega} \approx 0
\end{aligned}
$$

Thus,

$$
\frac{\partial F}{\partial \omega}=\left(\frac{\pi}{a}\right)^{2} \cdot \frac{\partial}{\partial \omega}\left(\frac{1}{\mu_{z}}\right)-2 \omega \varepsilon_{t}=-2 \omega \varepsilon_{t}
$$

Hence, $\varepsilon_{t}$ can be obtained from the numerical calculation of $\partial F / \partial \omega$.

Accordingly, the parameters $\varepsilon_{t}$ and $\mu_{z}$ are calculated as follows.

$$
\begin{gathered}
\varepsilon_{t}=-\frac{1}{2 \omega} \cdot \frac{\partial F}{\partial \omega} \\
\mu_{z}=\frac{\left(\frac{\pi}{a}\right)^{2}}{F-\frac{\omega}{2} \cdot \frac{\partial F}{\partial \omega}}
\end{gathered}
$$




\section{Validity of this method}

The validity of this method is confirmed by using simulation data. First, a dielectric sample with $\varepsilon_{t}$ of $4.2, \mu_{t}$ of $0.9, \mu_{z}$ of 0.4 is given in the waveguide for the simulation. Then S-parameters are calculated using HFSS. After that, the present method is applied for estimating the material parameters. Both results are shown in Fig. 2 with good agreement. Thus, the present method can estimate the parameters.

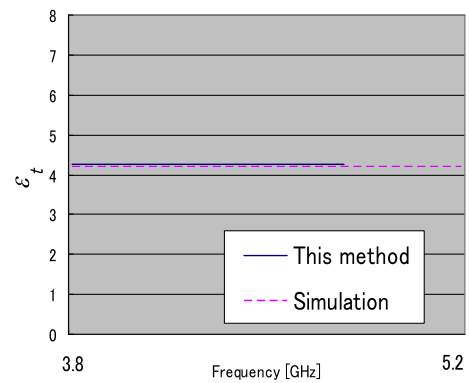

(a)

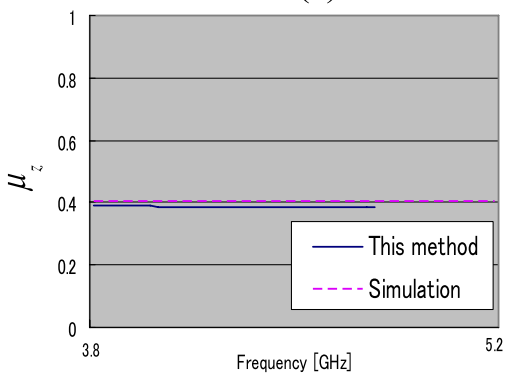

(c)

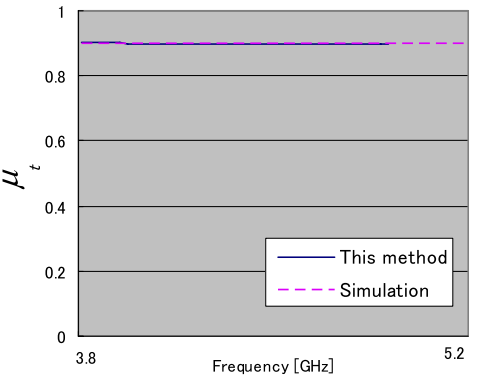

(b)

Fig. 2. Validity confirmation of this method (a) Transverse permittivity, (b) Transverse permeability, (c) Permeability along z-axis

\section{Comparison with two-waveguide method}

Separation of the parameters is also possible by using two waveguides with different widths $a$, where $a$ is $58.1 \mathrm{~mm}$ for WRJ-4 for $2.58-5.16 \mathrm{GHz}$ and $a$ is $40.0 \mathrm{~mm}$ for WRJ-6 for 3.75-7.50 GHz [2]. As for the two-waveguide method, the parameters are separated by using different widths $a$ in eq. (26). The results are explained below.

Fig. 3 shows the measured results. The present work uses only WRJ-6. On the other hand, the two-waveguide method by ref. [2] uses both WRJ-4 and WRJ-6. The both values show good agreement for transverse permittivity shown in Fig. 3 (a). The measured result by this work is a little bit noisy due to taking differentials on frequency. The measured results of permeability include a small error. It is due to the tolerance of the measurement setup. The actual cut-off frequency is a little bit higher than the nominal value. Then, the width $a$ is reduced by $1.5 \%$ for the calculation. The calibrated results are shown in Fig. 4. Two values in the red circles show good 


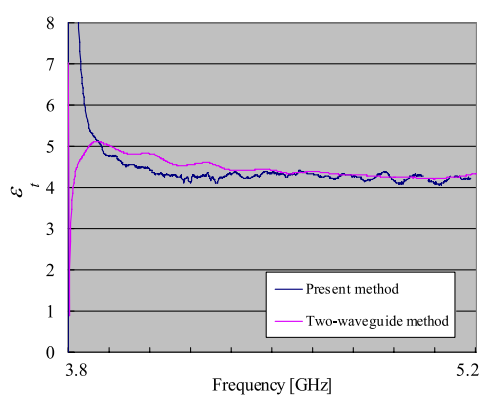

(a)

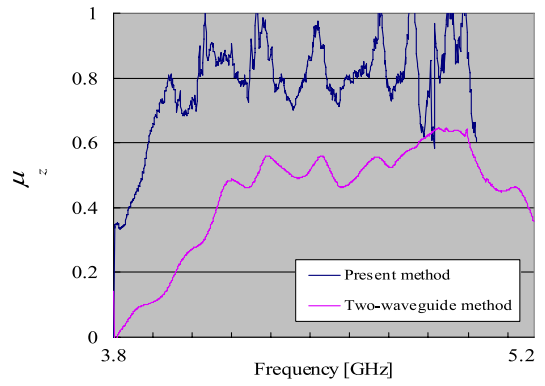

(c)

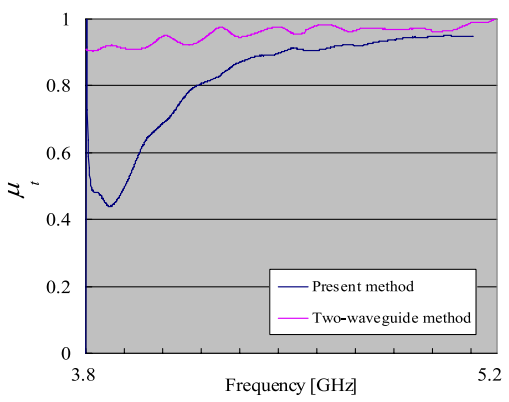

(b)

Fig. 3. Measured results (a) Transverse permittivity, (b) Transverse permeability, (c) Permeability along z-axis

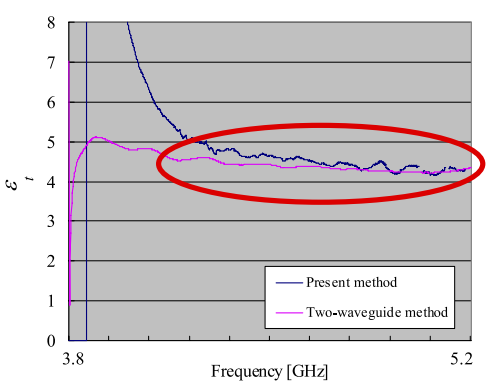

(a)

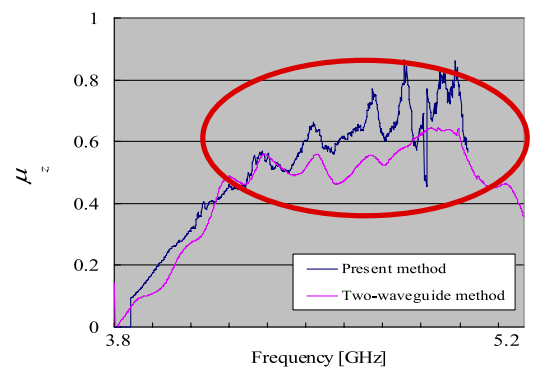

(c)

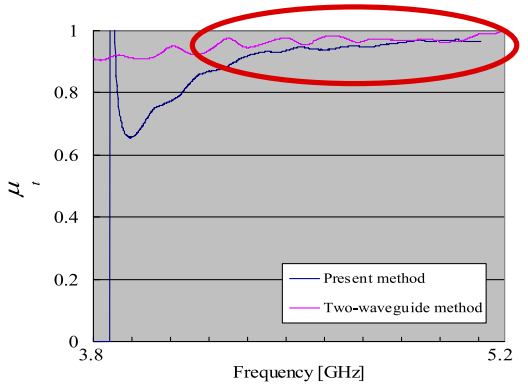

(b)

Fig. 4. Calibrated results (a) Transverse permittivity, (b) Transverse permeability, (c) Permeability along z-axis

agreement in this case except for the near cut-off frequency band, where the assumptions of eqs. (27), (28), and (29) are no longer valid.

Thus, the validity is confirmed by two different measurement methods based on different principles. Our measurement method has the great 
advantages of that we need only one type of waveguide and just one piece of sample. Thus, it is easier for us to measure the uniaxially anisotropic artificial dielectric by this novel method.

\section{Conclusion}

A novel measurement method for uniaxially anisotropic artificial dielectric was proposed. The measurements were carried out by the present method and two-waveguide method. The validity of the present method was confirmed by these measurements comparing with the two-waveguide method. Also simulation results support the validity of the present method.

Our meaurement procedure is extremely easier. Only one type of waveguide and just one piece of sample are required for the measurement. The calibrated result shows very good agreement with that of the two-waveguide method except for the near cut-off frequency band. Thus, the validity of the present method is confirmed, because they are calculated from quite different guiding priciples. The accuracy depending on the cut-off frequency is a remaining subject and it is for the future work. 